\title{
Financial hardship in metastatic colorectal cancer patients
}

\author{
Veena Shankaran* \\ *University of Washington, Department of Medicine, Division of Oncology, Seattle, WA, USA
}

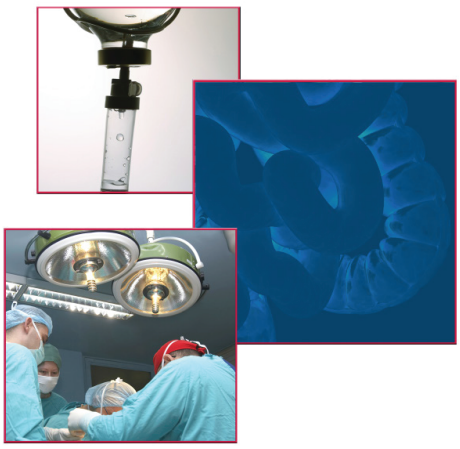
"cancer patients are at a significantly higher risk for financial hardship than people with other chronic diseases."

First draft submitted: 27 July 2020; Accepted for publication: 27 July 2020; Published online:

21 October 2020

Keywords: colorectal cancer $\bullet$ financial hardship $\bullet$ financial toxicity $\bullet$ metastasis $\bullet$ treatment

\section{Please introduce yourself \& provide a summary of your career to date}

My name is Veena Shankaran and I am a gastrointestinal medical oncologist at the Seattle Cancer Care Alliance (WA, USA). I am an Associate Professor of Medical Oncology at the University of Washington and Co-Director of the Hutchinson Institute for Cancer Outcomes Research at the Fred Hutchinson Cancer Research Center in Seattle (both WA, USA). I am a clinician investigator, so I spend a portion of my time taking care of patients with gastrointestinal cancers, and the rest of my time conducting research around health economics and health services. I have been interested in the topic of financial toxicity for many years, and this has been my primary research focus.

\section{Your abstract presented at ASCO 2020 looked at major financial hardship in metastatic colorectal cancer patients. Please could you explain this research in more detail \& explain the importance of this research?}

Many prior studies, including those from our group have demonstrated that a significant portion of people face some form of financial hardship during the course of cancer treatment and that cancer patients are at a significantly higher risk for financial hardship than people with other chronic diseases. Most of the prior studies have been retrospective, have included patients with a variety of different cancers and have relied primarily on survey data. We wanted to address some of these limitations by conducting a prospective longitudinal study to better understand how and when these financial hardships accumulate using both self-reported and objective financial measures.

We enrolled patients with metastatic colorectal cancers and their designated primary caregivers (caregiver participation optional). We administered surveys to patients every 3 months over a 12-month time period to assess the financial and employment impacts of cancer diagnosis; we obtained credit reports every 6 months.

We defined major financial hardships in the study as self-report of one of the following, accumulation of debts, a $20 \%$ of greater decline in income, taking loans to pay for cancer treatment or selling/refinancing ones primary home. What we observed was that an increasing proportion of patients experienced financial hardship over time, culminating with nearly $75 \%$ of patients reporting major financial hardship at 12 months [1].

\section{Interestingly, approximately $97 \%$ of your patients had insurance yet your study} demonstrates at 12 months, the incidence of patients who have sold their home \& were in debt was $3.6 \& 56.7 \%$, respectively. Why do you think this is?

Health insurance coverage clearly does not insulate or protect people from the devastating financial effects of cancer. First, most people with insurance still have significant deductibles or coinsurance they need to pay. Next, there are many other extra nonmedical costs such as food, transportation and childcare that families need to cover in order to pursue care. Finally, families have other fixed expenses (e.g., rent, mortgage) that still must be paid even as new cancer-related costs emerge. I think this study exposes a major problem with the way insurance is designed and delivered in this country. If the vast majority of people with cancer can suffer major financial hardships when 
going through treatment, then I think it says a lot - out-of-pocket costs, cost sharing, deductibles and copays are financially debilitating for most insured people.

In addition, insurance is tied to employment in this country and retaining insurance if you lose your job can be very expensive. For patients who either do not/cannot work or lose their job, there may be significant financial, emotional and logistic challenges to maintaining insurance coverage.

\section{Do you believe that the distress caused by financial hardship could be affecting patient overall survival \&/or quality of life (QoL)?}

Yes, I do. Other studies have demonstrated a correlation between financial hardship and poorer treatment adherence and even worse survival. In a prior study from our research group at the Hutchinson Institute for Cancer Outcomes Research, we demonstrated that cancer patients in Washington state who filed for bankruptcy after diagnosis were at higher risk of death than those patients who did not file. In this study, we will be reporting the association between financial burden and QoL. Our hypothesis is that patients who experience declines in their financial status also experience significant deterioration in their QoL.

\section{In your conclusion, you stated that clinical \& policy interventions are needed to protect cancer patients from financial hardship. If you could create \& implement policy, what would you put in place to protect cancer patients from this financial devastation?}

I think there are many potential solutions at the policy level but also at the clinical level. Recent studies have shown that the Affordable Care Act, for example, has improved the access and care for patients, particularly racial minorities. There has been discussion around value-based insurance design or value-based formulary, which I think are very interesting proposals to decrease out-of-pocket spending for interventions that are highly evidence-based.

I have been working for years to develop financial navigation program that brings together community-based organizations to provide financial counseling and support to patients' newly diagnosed with cancer and their caregivers. We are planning to launch a randomized study through SWOG (OR, USA) within the NCI Community Oncology Research Program (WA, USA) in 2021, which will test the impact of this financial navigation program on outcomes like QoL, financial hardship and caregiver burden, adherence to therapy and health utilization. Our hope is that these types of interventions at the clinic level can help to mitigate financial hardship in newly diagnosed cancer patients.

Financial \& competing interests disclosure

The author has no relevant affiliations or financial involvement with any organization or entity with a financial interest in or financial conflict with the subject matter or materials discussed in the manuscript. This includes employment, consultancies, honoraria, stock ownership or options, expert testimony, grants or patents received or pending, or royalties.

No writing assistance was utilized in the production of this manuscript.

Disclaimer

The opinions expressed in this interview are those of interviewee and do not necessarily reflect the views of Future Medicine Ltd.

\section{Open Access}

This work is licensed under the Attribution-NonCommercial-NoDerivatives 4.0 Unported License. To view a copy of this license, visit http://creativecommons.org/licenses/by-nc-nd/4.0/

\section{Reference}

1 Shankaran V, Unger JM, Darke A et al. Cumulative incidence of financial hardship in metastatic colorectal cancer patients: primary endpoint results for SWOG S1417CD. J. Clin. Oncol. 30(Suppl. 15), 7010

(2020). https://ascopubs.org/doi/abs/10.1200/JCO.2020.38.15_suppl.7010?af =R 